\title{
RELIABILITY AND VALIDITY OF THE QUALITY OF LIFE-ALZHEIMER'S DISEASE QUESTIONNAIRE IN MALAY LANGUAGE FOR MALAYSIAN OLDER ADULT WITH DEMENTIA
}

\author{
Kwai Ching Kan ${ }^{1}$, Ponnusamy Subramaniam ${ }^{1 *}$, Rosdinom Razali² and Shazli Ezzat Ghazali ${ }^{1}$ \\ ${ }^{1}$ Health Psychology Programme, School of Healthcare Sciences, Faculty of Health Sciences, Universiti Kebangsaan \\ Malaysia, 50300 Kuala Lumpur, Malaysia \\ ${ }^{2}$ Department of Psychiatry, Faculty of Medicine, Universiti Kebangsaan Malaysia Medical Centre, 56000 Kuala Lumpur, \\ Malaysia
}

*Corresponding author: Ponnusamy Subramaniam

Email: ponnusaami@ukm.edu.my

\begin{abstract}
The Quality of Life-Alzheimer's Disease (QOL-AD) questionnaire is a reliable, valid and popular outcome measure in dementia related studies. However, to date there is no published report on adopted version of QOL-AD in Malay language. Thus this preliminary study aimed to evaluate the reliability and validity of the QOL-AD in Malay language for patients with dementia and to examine the level of agreement between patient-caregiver as a proxy reports on patient's quality of life. A total of 65 patients with mild to moderate dementia and their caregivers were recruited from the psycho-geriatric clinic, Universiti Kebangsaan Malaysia Medical Centre using a cross sectional study design. The QOL-AD was translated into Malay language using the standard guidelines for cross-cultural adaptation of measure. The patient-caregiver dyads completed the QOL-AD in Malay language separately. Test-retest and internal consistency was evaluated for reliability and construct validity was tested with principal component factor analysis. The reliability of QOL-AD in Malay language was good with Cronbach alpha coefficients of 0.82 and 0.79 and intraclass correlation coefficients of 0.77 and 0.89 for patients and caregivers report respectively. Meanwhile a positive moderate agreement was achieved between patient and caregiver rating $(r=0.60, p<0.01)$. The principal component factor analysis extracted 4 factors for both patient and caregiver reports which explained 66.02\% (patients' report) and 64.24\% (caregivers' report) of the total variance of the scale. As a conclusion, the QOL-AD in Malay language is a reliable and valid tool to measure the quality of life of people with mild to moderate dementia.
\end{abstract}

Keywords: Quality of life, dementia, caregiver, factor analysis, Malay language

\section{INTRODUCTION}

Dementia is highly associated with advanced age although it is not a normal part of ageing. Globally the prevalence of dementia doubles with every five-year increment in age after 65 years old. It is estimated that the world's population over the age of 60 will be 2 billion by 2050, an increase from 900 million in 2015 and population ageing is occurring rapidly in low- and middle-income countries (LMIC) which by 2050, an estimate of $80 \%$ of older adults will live in $\mathrm{LMIC}^{1}$. Malaysia population is undergoing demographic transition. It is projected Malaysia's population will increase from 28.6 million in 2010 to 41.5 million in 2040. In 2010, the elderly aged 65 and above is accounted for $5.0 \%$ of the total population and it will increase to $14.5 \%$ by $2040^{2}$. Hence dementia will be a public health priority in worldwide including LMIC like Malaysia.

Improving the quality of life (QOL) of people with dementia has become an important aspect in dementia care. Researchers start to recognize that QOL should be the primary measure in the intervention or management of dementia ${ }^{3-5}$ since there is still no breakthrough in curing the disease. There is emerging evidence that nonpharmacology intervention should be incorporated in helping people with dementia6, 7 with the aim to alleviate the behavioral problems, improve cognitive function and delay deterioration of the disease.

Several instruments of QOL had been developed based on Lawton ${ }^{8}$ theoretical framework in measuring the QOL of people with dementia?. Lawton $^{8}$ highlighted the important of multidimensionality in evaluating the $\mathrm{QOL}$ in older people and both subjective and objective dimension must be assessed together. This framework encompasses four main components including behavioral competence, generalized psychological well-being, environment and perceived QOL which are overlapping and interdependent ${ }^{8}$. Nonetheless, the approaches in administering these instruments whether through direct self-report, caregiver proxy report or direct observation assessment in people with dementia are at constant scrutiny ${ }^{10}$. The validity of self-reported QOL by people with dementia is still debatable due to the progressive decline of their global cognitive impairment 11,12 . However new findings showed that Quality of LifeAlzheimer's Disease questionnaire structure is 
easy to understand and support interview-based format $^{13}$. Thus allow people with mild to moderate dementia to provide reliable and valid responses pertaining to their own QOL directly.

The Quality of Life-Alzheimer's Disease (QOL-AD) scale was developed by Logsdon et al. ${ }^{14}$ based on Lawton's model. It is recommended by European consensus ${ }^{3}$ as the measure of choice for psychosocial study related to QOL for people with dementia. It has been widely used in both cross sectional ${ }^{11,15}$ and intervention studies ${ }^{16-20}$. It consists of five domains: interpersonal, functional, physical, psychological and environmental. This scale is appraised directly by both the person with dementia and caregivers. It has satisfactory reliability and validity but only applicable to participants with Mini-Mental State Examination scores of 10 and above ${ }^{14}$. This scale has been widely used, translated and validated into different languages including Japanese ${ }^{21}$, Mandarin ${ }^{22}$, Spanish ${ }^{23}$, French ${ }^{24}$ and Thai ${ }^{25}$.

However, to the best of our search, the validation of a Malay language of QOL-AD has not been established. Thus the current work aims to evaluate the reliability and validity of the QOL$A D$ in Malay language for people with dementia and their caregiver.

\section{METHODS}

We conducted a cross-sectional study from August till December 2016 in the Psycho Geriatric Clinic, Department of Psychiatry, Universiti Kebangsaan Malaysia Medical Centre. All participants were recruited from the psychogeriatric clinic based on the following criteria:

\section{Inclusion Criteria}

- Patient aged 60 years old and above, ambulatory and community dwelling with an active caregiver who lived with them or at least spent the daytime with them.

- Patient had a diagnosis of dementia or mild cognitive impairment made by a geriatric psychiatrist according to DSM-5 with MMSE score of 10 and above.

- $\quad$ Both the patient and caregiver were able to speak, read and write in Malay language.

- Caregiver was willing to give written consent to participate.

\section{Exclusion Criteria}

- A history of psychosis based on medical record, presence of psychiatric diagnosis (based on DSM-5) other than dementia (Major Neurocognitive Disorders), severe physical condition or uncorrected sensory problem e.g. vision or hearing.

\section{Sampling Method}

The sample size is calculated based on Gorsuch's rule of 5 suggestion on subjects-to-variable ratio 26. The total items in this scale is multiplied by 5 and hence the required sample size for this study was 65. Convenient sampling was used in this study. Both patient and the caregiver was informed and explained about the purpose and procedure of the study and written informed consent was obtained before the interview begin. A face-to-face interview was conducted with patient to complete the QOL-AD questionnaire. Meanwhile patient's caregiver completed the QOL-AD questionnaire as a proxy. This study was approved by the UKM Research Ethics Committee, Universiti Kebangsaan Malaysia.

\section{Measures \\ Mini-Mental State Examination (MMSE) Malay Version $^{27}$ \\ MMSE is a tool commonly used to screen cognitive impairment including for people with dementia ${ }^{28}$. It measures five domains of cognitive function: orientation, registration, attention and calculation, recall and language. The score ranges from 0 to 30 where higher scores indicate better cognition. The Malay version of MMSE was validated in local setting with modification on the test of attention and repetition of question. The MMSE Malay version is a valid and reliable screening tool for dementia among Malaysian population ${ }^{27}$.}

Quality of Life-Alzheimer's Disease (QOL-AD) ${ }^{14}$ The QOL-AD is a brief, 13-items scale designed to measure the patient's quality of life from both the patient and caregiver perspective. It uses simple and straightforward language and responses and includes assessments of the individual's relationships with friends and family, concerns about finances, physical condition, mood, and an overall view of quality of life. The items are scored from " 1 " being poor to " 4 " being excellent. The total score ranges from 13 to 52 with lower score indicates poorer quality of life. The total scores for patient and caregiver was calculated separately. A single weighted composite QOL-AD score is calculated by multiplying the patient score by 2 , adding the caregiver score and dividing the sum by 3 . This composite score weights the patient rating more heavily than the caregiver rating. This scale is a valid and reliable measure for individual with MMSE score of 10 or higher.

\section{Translation of the QOL-AD}

The original English version of the QOL-AD was translated into Malay language following the Mapi Research Trust translation guidelines ${ }^{29}$. First the original version of QOL-AD was translated into Malay language by two local professional independant translators. These two forwards translations were then compared and discussed by both the translators and researchers 
and a reconcile version was produced. In the case of discrepancy, the original author would be contacted for clarification on the reconcile version and modification should be made accordingly. The reconcile QOL-AD in Malay language was back translated by a bilingual professional translator who was blinded to the original version of the questionanaire. The backward translated version was revised by a committee panel comprising of psychogeriatrician, clinical psychologist and neurologist to fine tune the translation and ensure the content validity, cultural and linguistic appropriateness. A pilot study involved 10 Malay-speaking patients with mild to moderate dementia and their caregivers was conducted. The feedback and comment obtained from the pilot study was discussed and reviewed by the researchers and appropriate amendments were rectified and the final version of QOL-AD in Malay language was ready for psychometric assessment.

\section{Statistical Analysis}

Reliability was evaluated by testing the correlation of each item with the total QOL-AD score and the correlation between scale items and the general item-13 "Life as a whole" for both patient and caregiver report versions. Cronbach a coefficient was used to determine the internal consistency of the QOL-AD scale for both patient and caregiver reports. Cronbach a coefficient of $\geq 0.70$ indicates a satisfactory internal consistency. Correlation between patient and caregiver reports for each item and total score was used to examine the agreement between both parties rating on quality of life. Test-retest reliability was assessed after two weeks interval. Intraclass correlation coeffiecients (ICC) was used to evaluate the agreement. The ICC $<0.40$ is considered as poor agreement, 0.41 to 0.60 is moderate agreement, 0.61 to 0.80 is good agreement and $>0.80$ is excellent agreement ${ }^{30}$.

Construct validity was examined through principal component factor analysis with varimax rotation on both patient and caregiver reports. Kaiser-Meyer-Olkin (KMO) and Bartlett's test of sphericity were used to test for the appropriateness of factor analysis. The eigenvalue great than 1 was used to determine the number of factors to extract.

\section{RESULTS}

\section{Participants' Characteristic}

Table 1 describes the socio-demographic characteristic of the participants. A total of 65 patients were recruited in this study. The patients age ranged from 62 to 89 with the mean/sd of $77.37 \pm 6.67$. MMSE score ranged from $10-25$ with its mean/sd of $18.12 \pm 4.32$. Sixty percent of the patients were female. The largest ethnic group (53.8\%) were Malay, followed by Chinese (36.9\%) and Indians (9.2\%). For marital status, almost half of them were married $(49.2 \%)$ and remaining half were widows (47.7\%). In term of education, half of the patients $(52.3 \%)$ studied up to primary level, $38.5 \%$ into secondary and 9.2\% obtained a diploma or higher education. Almost half $(47.7 \%)$ of the patients were diagnosed with Alzheimer's disease, $36.9 \%$ had vascular type of dementia, $9.2 \%$ in mild cognitive impairment group and the remaining $6.2 \%$ with parkinson type of dementia. Majority of the patients' caregivers were their children (66.2\%) and followed by spouse (26.2\%).

Table 1: Socio Demographic Characteristic of Participants

\begin{tabular}{|c|c|c|c|}
\hline Variables & $\mathrm{N}=65$ & n (\%) & Mean/SD \\
\hline Age & & & $77.37 \pm 6.67$ \\
\hline MMSE & & & $18.12 \pm 4.32$ \\
\hline \multirow[t]{2}{*}{ Gender } & Male & $26(40.0)$ & \\
\hline & Female & $39(60.0)$ & \\
\hline \multirow[t]{3}{*}{ Race } & Malay & $35(53.8)$ & \\
\hline & Chinese & $24(36.9)$ & \\
\hline & Indian & $6(9.2)$ & \\
\hline \multirow[t]{3}{*}{ Marital Status } & Married & $32(49.2)$ & \\
\hline & Widow & 31 (47.7) & \\
\hline & Single & $2(3.1)$ & \\
\hline \multirow[t]{3}{*}{ Education Level } & Primary & $34(52.3)$ & \\
\hline & Secondary & $25(38.5)$ & \\
\hline & Diploma and above & $6(9.2)$ & \\
\hline \multirow{4}{*}{ Type of Dementia } & Alzheimer & 31 (47.7) & \\
\hline & Vascular & 24 (36.9) & \\
\hline & Parkinson & $4(6.2)$ & \\
\hline & Mild Cognitive Impairment & $6(9.2)$ & \\
\hline \multirow[t]{4}{*}{ Caregiver Relationship } & Spouse & $17(26.2)$ & \\
\hline & Children & $43(66.2)$ & \\
\hline & Grandchildren & $2(3.1)$ & \\
\hline & Sibling & $3(4.6)$ & \\
\hline
\end{tabular}




\section{Reliability of the QOL-AD in Malay language}

Table 2 presents the internal consistency of the QOL-AD. Item-total correlation ranged from 0.41 to 0.74 for patients and 0.19 to 0.77 for caregivers. However, the correlations of each item with item-13 "life as a whole" were lower in both the patients $(r=0.19-0.65)$ and caregivers $(r=0.04-0.53)$. Overall Cronbach alpha coefficient levels for both patient and caregiver reports were well within the acceptable range $(0.82$ and 0.79$)$ which indicated good internal consistency.

Spearman's Rho correlation analysis showed that patients and caregivers achieved moderate agreement on items related to physical health $\left(r_{s}\right.$ $=0.53)$, energy $\left(r_{s}=0.50\right)$, marriage $\left(r_{s}=0.57\right)$, friends $\left(r_{s}=0.57\right)$, ability to do chores $\left(r_{s}=0.51\right)$, money $\left(r_{s}=0.57\right)$ and life as a whole $\left(r_{s}=0.51\right)$. Weak correlations were observed on items related to mood, memory, living situation, family and self as a whole. Whereas there was no significant correlation observed on item ability to do things for fun between both parties.

Test-retest reliability was measured on a subset of 15 patient-caregiver dyads at two week interval. Intra-class correlation coefficients were within the acceptable range (ICC $=0.77$ for patients and 0.89 for caregivers).

Table 2: Item-Total Correlation and Coefficient Alpha of the Quality of Life-AD Scale

\begin{tabular}{|c|c|c|c|c|c|}
\hline \multirow[t]{2}{*}{ Item } & \multicolumn{2}{|c|}{ Patient Correlation } & \multicolumn{2}{|c|}{ Caregiver Correlation } & \multirow{2}{*}{$\begin{array}{l}\text { Patient- } \\
\text { caregiver } \\
\text { correlation }\end{array}$} \\
\hline & Total & "Life as a Whole" & Total & "Life as a Whole" & \\
\hline 1.Physical health & $0.59^{* *}$ & $0.31^{*}$ & $0.56^{* *}$ & $0.41^{* *}$ & $0.53^{* *}$ \\
\hline 2. Energy & $0.69^{* *}$ & $0.33^{* *}$ & $0.59^{* *}$ & $0.31^{*}$ & $0.50^{* *}$ \\
\hline 3. Mood & $0.61^{* *}$ & $0.31^{*}$ & $0.55^{* *}$ & $0.35^{* *}$ & $0.32^{*}$ \\
\hline 4. Living situation & $0.50^{* *}$ & $0.31^{*}$ & 0.19 & 0.14 & $0.29^{*}$ \\
\hline 5. Memory & $0.42^{* *}$ & 0.19 & $0.26^{*}$ & 0.04 & $0.26^{*}$ \\
\hline 6. Family & $0.42^{* *}$ & $0.31^{*}$ & $0.43^{* *}$ & 0.19 & $0.29^{*}$ \\
\hline 7. Marriage & $0.41^{* *}$ & $0.46^{* *}$ & $0.58^{* *}$ & $0.46^{* *}$ & $0.57^{* *}$ \\
\hline 8. Friends & $0.51^{* *}$ & $0.42^{* *}$ & $0.59^{* *}$ & $0.36^{* *}$ & $0.57^{* *}$ \\
\hline 9. Self as a whole & $0.73^{* *}$ & $0.65^{* *}$ & $0.77^{* *}$ & $0.53^{* *}$ & $0.32^{* *}$ \\
\hline $\begin{array}{l}\text { 10.Ability to do chores around } \\
\text { the house }\end{array}$ & $0.57^{* *}$ & $0.42^{* *}$ & $0.61^{* *}$ & $0.44^{* *}$ & $0.51^{* *}$ \\
\hline 11. Ability to do things for fun & $0.57^{* *}$ & $0.42^{* *}$ & $0.57^{* *}$ & $0.40^{* *}$ & 0.23 \\
\hline 12. Money & $0.59^{* *}$ & $0.43^{* *}$ & $0.44^{* *}$ & $0.32^{*}$ & $0.57^{* *}$ \\
\hline 13. Life as a Whole & $0.74^{* *}$ & & $0.71^{* *}$ & & $0.51^{* *}$ \\
\hline Total correlation ${ }^{\mathrm{b}}$ & & & & & $0.60^{* *}$ \\
\hline Coefficient Alpha & 0.82 & & 0.79 & & \\
\hline
\end{tabular}

${ }^{a}$ Correlations are based on Spearman's Rho, ${ }^{b}$ Pearson correlation, ${ }^{*} p<0.05,{ }^{* *} p<0.01$

Validity of the QOL-AD Malay Language

The construct validity using KMO test values were 0.76 for patients' report and 0.69 for caregivers' report. Barlett's test of sphericity of both reports were statistically significant $(p<0.001)$. Both of this data ensured the adequacy for factor analysis. Four factors were extracted from the 13 items scale for both patients' and caregivers' reports when eigenvalue was greater than 1 . Patients' report explained $66.02 \%$ of the total variance whereas caregivers' report explained $64.24 \%$ of the total variance.

Table 3 shows the loading of the items on four extracted factor after rotated by varimax method. For the patient's report, factor 1 was loaded by three items (physical health, energy and memory) that was corresponded to health factor. Three items loaded on factor 2 (mood, friends and ability to do chores) was best categorized under the social wellbeing and functional domain. Factor 3 was also loaded with three items (living situation, family and money) reflecting on the social-environment factor whereas factor 4 loaded with four items (marriage, self as a whole, ability to do things for fun and life as a whole) was best described as overall psychological wellbeing. As for the caregiver's report, factor 1 was loaded with five items (physical health, energy, mood, self as a whole and life as a whole) representing the physical and psychological wellbeing. Factor 2 described on the social wellbeing domain was loaded with three items (memory, friends and ability to do things for fun). Similarly factor 3 also had three items (living situation, family and marriage) representing the environment and close interpersonal relationships and lastly two items (ability to do things for fun and money) were loaded on factor 4 representing the functional domain. 
Malaysian Journal of Public Health Medicine 2019, Vol. 19 (1): 56-63

Table 3: QOL-AD Malay Language items loading on extracted factors after varimax rotation

\begin{tabular}{|c|c|c|c|c|c|c|c|c|c|}
\hline \multirow[t]{2}{*}{ Items } & & \multicolumn{4}{|c|}{ Patients' factor } & \multicolumn{4}{|c|}{ Caregivers' factor } \\
\hline & & 1 & 2 & 3 & 4 & 1 & 2 & 3 & 4 \\
\hline 1. & Physical health & 0.76 & & & & 0.87 & & & \\
\hline 2. & Energy & 0.76 & & & & 0.81 & & & \\
\hline 3. & Mood & & 0.80 & & & 0.53 & & & \\
\hline 4. & Living situation & & & 0.76 & & & & 0.74 & \\
\hline 5. & Memory & 0.72 & & & & & 0.69 & & \\
\hline 6. & Family & & & 0.77 & & & & 0.79 & \\
\hline 7. & Marriage & & & & 0.64 & & & 0.71 & \\
\hline 8. & Friends & & 0.70 & & & & 0.73 & & \\
\hline 9. & Self as a whole & & & & 0.57 & 0.58 & & & \\
\hline 10. & Ability to do chores & & 0.65 & & & & & & 0.68 \\
\hline 11. & $\begin{array}{l}\text { Ability to do things } \\
\text { for fun }\end{array}$ & & & & 0.69 & & 0.66 & & \\
\hline 12. & Money & & & 0.59 & & & & & 0.84 \\
\hline 13. & Life as a whole & & & & 0.69 & 0.45 & & & \\
\hline
\end{tabular}

\section{DISCUSSION}

The aim of this study was to examine the reliability and validity of QOL-AD in Malay language among people with dementia and their primary caregiver. In general, the QOL-AD in Malay language was a reliable scale with good internal consistency for both the patientinterview version and caregiver-questionnaire version.

The present study indicates that QOL-AD in Malay language is easy to understand and comprehend although majority of the patients only obtained up to an elementary education level. This is parallel with original recommendation that $\mathrm{QOL}$ questionnaire should be structured in straightforward, simple and supportive interview-based format ${ }^{8,}{ }^{14}$. Also, current finding is consistent with the Chinese version of QOL-AD validation $^{31}$ reporting that participants with dementia with lower education level were capable to reliably and validly rate their own quality of life. Moreover, this study also supports the original version of QOL-AD ${ }^{14}$ on the feasibility in self-reporting quality of life by people with mild to moderate dementia with MMSE score of 10 or higher.

This study demonstrated a moderate patientcaregiver (proxy) agreement for the QOL-AD in Malay language. However the patient-caregiver agreement according to items in QOL-AD was varied in different studies ${ }^{31}, 32$. This could be due to cultural and socio differences between countries. For example, most of the studies had indicated that people with dementia rated their "functional abilities" higher than their caregivers rating ${ }^{33,}{ }^{34}$ but in this study the item "ability to do chores" showed moderate agreement in both patient and caregiver rating. This may due to the traditional value of supporting the elderly by family members is still preserved in rural area ${ }^{35}$ and for urban area the engagement of domestic helpers in easing the household chores could have led to no observational differences in this item. The item "ability to do things for fun" was consistently appeared to be not significantly correlated between the patients and caregivers agreement ${ }^{14,32}$. It is important to note that despite patient and caregiver reports are in moderate agreement but their perception about quality of life is not identical. This once again showed the involvement of subjective aspect in assessing quality of life. This correlation most likely reflects the real difference in the way how the patients themselves and their caregivers perceived the subjective components of $\mathrm{QOL}$ rather than a lack of reliability of the measure itself.

Factor analysis revealed the QOL-AD in Malay language extracted four factors for both patient and caregiver reports which are consistent with Shata et al., ${ }^{32}$ findings. In contrast, present findings were different from other studies ${ }^{23}, 36$ which only extracted three factors. Again, these differences may due to variation in study population and socio-demographical factors. For example, Revell et al. ${ }^{36}$ study included healthy participants with higher education level but for this and Shata et al., ${ }^{32}$ study, majority of the participants were of elementary education level. Education level has been reported as the only possible socio-demographic confounder in both patient and caregiver $\mathrm{QOL}$ rating $^{14}$. Sociodemographic factors may result in differences in perception of the subjective dimension of QOL.

In this study, the items loaded in each identified domains fitted better in the caregiver report than the patient report. This once again reflects on the differences between the patients' and caregivers' perception especially on the subjective aspect of QOL. Nonetheless, it is also important to note that the four domains theorized by Lawton ${ }^{8}$ are interdependent and overlapping which further explained the differences of items loading at each domain in this study. 
Few limitations need to be addressed in this study. This study was conducted in a single teaching hospital in city centre which may cause sample selection bias and this might not represent the whole Malay speaking population especially those residing in rural area. Based on present study inclusion criteria of the ability to speak, read and write in Malay language may exclude potential participants who are illiterate but able to speak and understand the language. Also, majority of the illiterate rural older adults would be left out. Moreover, the present study population only consisted of old community dweller and hence the generalisation of this scale to those living in nursing homes and residential facilities need to be done with caution. Besides current study only managed to recruit participants from the three main ethnics that failed to represent the nation multi ethnicities composition. Therefore, future validation study on QOL-AD in Malay language should consider current study limitations in order to provide a measure that can be generalisable for people with dementia in Malaysia.

\section{CONCLUSION}

In summary, this is the first validation study of the QOL-AD in Malay language for Malaysian older adults with dementia. This study demonstrates the QOL-AD in Malay language is a valid and reliable scale in evaluating the quality of life for people with mild to moderate dementia living in community. Besides the QOL$A D$ in Malay language is proven to be easy to use and supports both the interview based selfreport of people with dementia and the caregiver proxy questionnaire assessment.

\section{ACKNOWLEDGEMENTS}

Kwai Ching Kan's doctoral studies are jointly supported by 'MyBrain 15 Program' under Ministry of Higher Education Malaysia and Universiti Kebangsaan Malaysia's research grant GGPM-2013-046. The authors would like to thank Mapi Research Trust for granting the permission to validate QOL-AD in Malay language. We are very grateful for the participants and caregivers for their cooperation and generosity in time.

\section{REFERENCES}

1. World Health Organization and Alzheimer's Disease International Dementia: a public health priority 2012. https: / /www.who.int/mental_health/pu blications/dementia_report_2012/en/ (accessed 2 July 2017).

2.

$\begin{array}{lcr}\begin{array}{l}\text { Department } \\ \text { Population }\end{array} \quad \begin{array}{c}\text { Statistic } \\ \text { projection }\end{array} & \begin{array}{r}\text { Malaysia. } \\ \text { (revised), }\end{array} \\ \text { Malaysia, } & 2010-2040 . & 2017 . \\ \text { https://www.dosm.gov.my/ } & \text { (accessed 2 } \\ \text { July 2017). } & \end{array}$

3. Moniz-Cook E, Vernooij-Dassen M, Woods $\mathrm{R}$, et al. A European consensus on outcome measures for psychosocial intervention research in dementia care. Aging Ment Health. 2008; 12(1):14-29.

4. Yang $F$, Dawes $\mathrm{P}$, Leroi I, et al. Measurement tools of resource use and quality of life in clinical trials for dementia or cognitive impairment interventions: protocol for a scoping review. Systematic Reviews. 2017; 6(1):22.

5. Beerens HC, Zwakhalen SM, Verbeek H, et al. Factors associated with quality of life of people with dementia in long-term care facilities: a systematic review. Int J Nurs Stud. 2013; 50(9):1259-1270.

6. Cabrera E, Sutcliffe C, Verbeek H, et al. Non-pharmacological interventions as a best practice strategy in people with dementia living in nursing homes. A systematic review. European Geriatric Medicine. 2015; 6(2):134-150.

7. Takeda M, Tanaka T, Okochi M, et al. Non-pharmacological intervention for dementia patients. Psychiatry and Clinical Neurosciences. 2012; 66(1):1-7.

8. Lawton MP. Assessing quality of life in Alzheimer disease research. Alzheimer Dis Assoc Disord. 1997; 11(Suppl 6):9199.

9. Ready RE, Ott BR. Quality of Life measures for dementia. Health Qual Life Outcomes. 2003; 1:11.

10. Naglie G. Quality of life in dementia. Can J Neurol Sci. 2007; 34(Suppl 1):S57-61.

11. Woods RT, Nelis SM, Martyr A, et al. What contributes to a good quality of life in early dementia? awareness and the QoL-AD: a cross-sectional study. Health and Quality of Life Outcomes. 2014; 12:94-94.

12. Ready RE, Ott BR, Grace J. Insight and cognitive impairment: effects on qualityof-life reports from mild cognitive impairment and Alzheimer's disease patients. Am J Alzheimers Dis Other Demen. 2006; 21(4):242-248.

13. Logsdon RG, Gibbons LE, McCurry SM, et al. Assessing quality of life in older adults with cognitive impairment. Psychosom Med. 2002; 64(3):510-519.

14. Logsdon RG, Gibbons LE, McCurry SM, et al. Quality of life in Alzheimer's disease: 
patient and caregiver reports. Journal of Mental Health and Aging. 1999; 5:21-32.

15. Miguel S, Alvira $M$, Farré $M$, et al. Quality of life and associated factors in older people with dementia living in long-term institutional care and home care. European Geriatric Medicine. 2016; 7(4):346-351.

16. Charlesworth G, Burnell K, Crellin N, et al. Peer support and reminiscence therapy for people with dementia and their family carers: a factorial pragmatic randomised trial. Journal of Neurology, Neurosurgery \& Psychiatry. 2016; 87(11):1218-1228.

17. Subramaniam P, Woods B, Whitaker C. Life review and life story books for people with mild to moderate dementia: A randomised controlled trial. Aging \& Mental Health. 2014; 18(3):363-375.

18. O'Shea E, Devane D, Cooney A, et al. The impact of reminiscence on the quality of life of residents with dementia in longstay care. International Journal of Geriatric Psychiatry. 2014; 29(10):10621070.

19. Almeida OP, MacLeod C, Flicker L, et al. RAndomised controlled trial to imProve depresslon and the quality of life of people with Dementia using cognitive bias modification: RAPID study protocol. BMJ Open. 2014; 4(7):1-8.

20. Marshall A, Spreadbury J, Cheston R, et al. A pilot randomised controlled trial to compare changes in quality of life for participants with early diagnosis dementia who attend a 'Living Well with Dementia' group compared to waiting-list control. Aging Ment Health. 2015; 19(6):526-535.

21. Matsuia T, Nakaakia S, Murataa Y, et al. Determinants of the quality of life in Alzheimer's disease patients as assessed by the Japanese version of the quality of life - Alzheimer's disease scale. Dement Geriatr Cogn Dis. 2006; 21(3):182-91.

22. Lin Kiat Yap P, Yen Ni Goh J, Henderson $L M$, et al. How do Chinese patients with dementia rate their own quality of life? International Psychogeriatrics. 2008; 20(3):482-493.

23. Gómez-Gallego $M$, Gómez-Amor J, Gómez-García J. Validation of the Spanish version of the QoL-AD scale in Alzheimer disease patients, their carers, and health professionals. Neurología (English Edition). 2012; 27(1):4-10.

24. Wolak A, Novella JL, Drame M, et al. Transcultural adaptation and psychometric validation of a Frenchlanguage version of the QoL-AD. Aging Ment Health. 2009; 13(4):593-600.

25. Buasi N, Permsuwan U. Validation of the Thai QOL-AD version in Alzheimer's patients and caregivers. The Australasian Medical Journal. 2014; 7(6):251-259.

26. Gorsuch RL. Factor analysis (2nd ed). Erlbaum: Hillsdale,NJ; 1983.

27. Zarina ZA ZO, Che Wan AH. Validation of Malay Mini Mental State Examination. Malaysian Journal of Psychiatry. 2007; 16(1):16-19.

28. Folstein MF, Folstein SE, McHugh PR. "Mini-mental state". A practical method for grading the cognitive state of patients for the clinician. J Psychiatr Res. 1975; 12(3):189-198.

29. Mapi Research Trust. Linguistic validation guidance of a clinical outcome assessment (COA). https: / /mapigroup.com/services/langua ge-services/linguistic-validation/ (accessed 20 July 2016).

30. Shrout PE. Measurement reliability and agreement in psychiatry. Stat Methods Med Res 1998; 7(3):301-317.

31. $Y u$ HM, He RL, Ai YM, et al. Reliability and validity of the quality of lifeAlzheimer disease Chinese version. J Geriatr Psychiatry Neurol. 2013; 26(4):230-236.

32. Shata Z, El-Kady H, Ibrahim H. Reliability and Validity of an Arabic Version of Quality of life - Alzheimer Disease in Alexandria, Egypt. Int J Behav Res Psychol. 2017; 3(8):157-163.

33. Kiyak HA, Teri L, Borson S. Physical and Functional Health Assessment in Normal Aging and in Alzheimer's Disease: SelfReports vs Family Reports. The Gerontologist. 1994; 34(3):324-331.

34. Li M, Harris I, Lu ZK. Differences in proxy-reported and patient-reported outcomes: assessing health and functional status among medicare beneficiaries. BMC Medical Research Methodology. 2015; 15:62. 
35. Wan-Ibrahim WAZ, I. The availability of family support of rural elderly in Malaysia. World Applied Sciences Journal. 2014; 30(7): 899-902.

36. Revell AJ, Caskie GIL, Willis SL, et al. Factor structure and invariance of the quality of life in Alzheimer's disease (QoL-AD) scale. Experimental aging research. 2009; 35(2):250-267. 